\title{
Further evidence for positive correlations of intelligence and suicide rate across Italian regions: Responsum to Felice (2010)
}

\author{
Martin Voracek \\ University of Vienna \\ University of Göttingen
}

This manuscript version: June 9, 2013

Address correspondence to Martin Voracek, D.Sc., D.M.Sc., Ph.D., Department of Basic Psychological Research and Research Methods, School of Psychology, University of Vienna, Liebiggasse 5, Room 03-46, A-1010 Vienna, Austria, and Georg Elias Müller Department of Psychology, Georg August University of Göttingen, Goßlerstraße 14, D-37073 Göttingen, Germany, or e-mail (martin.voracek@univie.ac.at). 
Summary.--Voracek (2009) found regional IQ estimates and suicide rates to be positively correlated across 13 (out of 20) Italian regions. Felice (2010) criticized this group-level (ecologic) finding from geographic analysis on various grounds. These arguments are addressed and rebutted here. Further, replication tests with two new, independent data sources for regional Italian IQ, now including either 19 or all the 20 regions of Italy, show that these are similarly positively correlated with suicide rates. Finally, the group-level finding of Voracek (2009) and its two successful replications are now directly confirmed by recent individual-level (register-based) evidence for higher educational attainment (an IQ proxy) in Italian suicide victims, compared to deaths from all other causes. 
Voracek (2009), using data from Lynn (2010a), reported a positive ecologic (group-level, geographic) correlation between regional suicide rates and a proxy of regional IQ across 13 Italian regions with available data (out of a total of 20 regions). Both the source reference (Lynn, 2010a) and the research application (Voracek, 2009) of these IQ data have been criticized by the same commentator (Felice, 2010; Felice \& Giugliano, 2011) on various grounds.

The account of Lynn (2010a), particularly his evidence for north-south differences in regional Italian IQ, has been criticized by further commentators as well (Beraldo, 2010; Cornoldi, Belacchi, Giofrè, Martini, \& Tressoldi, 2010; Cornoldi, Giofré, \& Martini, 2013). A summary or indepth evaluation of these partly extensive, elaborate critiques is beyond the intended and needed scope of the present account and not central to it. As for one example, Beraldo (2010) stated that causal claims of Lynn (2010a) were insufficiently supported by the correlational evidence presented. It is however worth mentioning that Lynn (2010b, 2012a) in two detailed replies has addressed the gist of the arguments brought forward (particularly the view (Felice \& Giugliano, 2011) that in fact there are no north-south differences in IQ in Italy) and rebutted these with new data. Cornoldi, et al. (2013) showed that there still are north-south differences in Italian IQ estimates, but, according to 2006 vs. 2009 comparisons of the Programme for International Student Assessment (PISA) results, these north-south differences appear to decline, indicative of an increase in achievement among the southern Italian regions. Also, Lynn (2012b) has presented evidence for a similar north-south difference in regional IQ for another Mediterranean country (Spain).

Within this scholarly discussion and exchange, a novel line of inquiry is due to Rindermann, Woodley and Straford (2012). Bearing in mind that cross-national as well as intranational estimates of mean individual cognitive ability might be confounded with the regions' general developmental status, these researchers analyzed the geographic prevalence of configural genetic variation (i.e., haplotypes) found in the male Y chromosome. Such haplotypes can be taken as an evolutionary marker because genetic loci on the male sex chromosome provide unambiguous markers of 
(paternal) ancestry, are selection neutral, and do not recombine. Rindermann, et al. (2012) found that the prevalence of evolutionarily more recent (vs. older) Y chromosome haplotypes corresponded to higher (vs. lower) geographically based cognitive ability estimates. This finding emerged in a cross-national test as well as in two independent intranational tests. Of note, the regions compared for the latter two tests were those of Italy and Spain, for which corresponding north-south differences in IQ have been noted (Lynn, 2010a, 2012b).

Since the comment of Felice (2010) on Voracek (2009), important new data sources have become available. The main goal of this note is to utilize these recently published data, in order to revisit, re-evaluate, and replicate the data, evidence, and conclusions of Voracek (2009). Felice (2010) opted to entitle his comments on Voracek (2009) as a "reply"; hence, this note is a Responsum (cf. Wainer, 1989) to Felice (2010).

\section{OBJECTIONS AND MISPERCEPTIONS IN FELICE (2010)}

There appear to be three major objections to the approach and evidence of Voracek (2009), as listed in both the abstract and the introductory section of Felice (2010). First, Voracek (2009) did not use intelligence (IQ) data, but rather data from educational attainment tests; second, the statistical analyses were inadequate, given the small number of cases; and third, correlation does not imply causation.

Rebuttal of these points is straightforward, as follows. First, there is evidence that IQ and educational attainment are so highly positively correlated (with $r>.80$ ), as to be interchangeable for research purposes (Deary, Strand, Smith \& Fernandes, 2007; this key reference was already cited, among others, by Voracek (2009) but ignored by Felice (2010) in his argumentation). The second argument above is irrelevant for ecologic (total-population) research, as this approach already uses all available data units (i.e., the totality of evidence), regardless of their number. Of note in this context, Felice (2010) basically used the same data-analytic approach as Voracek (2009), the main difference perhaps being that his model, in his own words, is "weak, with too many variables and a 
high collinearity" (Felice, 2010, p. 733). As for the third argument above, this, of course, was never claimed and, in fact, is a commonplace, well-known and well-acknowledged among researchers.

In the remainder of his commentary (Felice, 2010, p. 735), three common misperceptions, misunderstandings, or allegations appear, pertaining to human intelligence and to what IQ tests measure, all of which demonstrably are factually false (as referenced below) and thus have no bearing on the evidence of Voracek (2009). First, IQ allegedly is not linked to attainment tests (for counter-evidence, see Deary, et al., 2007); second, IQ measures allegedly somehow are unreliable and invalid (see Snyderman \& Rothman, 1987, 1990, for expert consensus about the psychometric properties and the construct and predictive validity of IQ tests; see Hunt, 2011, and Deary, 2012, for up-to-date scholarly perspectives on human intelligence research); and third, heritability (of IQ or general) allegedly means immutability and determination (see Plomin, DeFries, McClearn \& McGuffin, 2008, for contemporary behavior genetic interpretation and the actual meaning of genetic effects).

Finally, Felice (2010) introduces latitude as an alternative explanatory variable for regional variation in suicide rates across Italy. He makes a great deal from the finding that, according to his ecologic correlational analysis, regional suicide rates correspond numerically slightly more closely to regions' latitude than to regions' IQ estimates (Pearson $r=.64$ vs. .57). However, this difference is, of course, nominally not significant and it is not meaningful within the framework of group-level analysis, and thus is an overinterpretation. In this context, Felice claims that "climatic differences are a well-established subject" (Felice, 2010, p. 733) in suicidology, but then provides only a single, remote, not widely cited, and dated reference (Ellner, 1977) for this claim. Contrary to Felice's assertion, climatic hypotheses and explanations for suicidal behavior originally flourished in suicidological accounts of the late 19th century (e.g., in the classical works of Durkheim, 1897/1997; Morselli, 1881, and others), but do not play an appreciable role in contemporary suicidology. It is 
also conspicuous that Felice (2010) did not provide any clear rationale why in this specific context latitude should be considered as an important explanatory variable.

The best empirical argument against this possibility is that, paralleling the evidence of Voracek (2009) for Italy, prior related research has shown similar positive ecologic associations between regional suicide rates and IQ estimates in intranational analyses of countries which, unlike Italy, lack a significant north-south extension of territory and population, such as Austria (Voracek, 2006b), Belarus (Voracek, 2005), Denmark (Voracek, 2006a), or the Netherlands (Kerkhof \& Kunst, 1994). As a consequence, the evidence from these countries excludes latitude as a confounder of observed associations between regional suicide rates and IQ and disconfirms an important explanatory role of latitude on its own.

More generally, it has been emphasized that the evidence for Italy, i.e., the group-level correlation between suicide rates and IQ estimates being positive, was "replicating the majority finding from prior related studies" (Voracek, 2009, p. 1023), with a considerable number of references provided for that statement. Apparently unimpressed by the cumulative research evidence, Felice (2010) opted to ignore this in his commentary altogether. It is however easy to see that such corroborating, convergent evidence from ecologic analyses of quite different countries and the repeated successful replication of hypothesized effects across such independent tests poses huge credibility and veridicality problems for any specific, localized, ad hoc, or post hoc explanations and speculations, such as those offered by Felice (2010). In addition, the evidence of Rindermann, et al. (2012) suggests that north-south differences in Italian (as well as in Spanish) IQ map onto known population genetic gradients within these countries; hence, correlations with latitude and, by implication, with climate are likely to be spurious and without substance.

\section{REPLICATIONS AND DIRECT CONFIRMATION OF VORACEK (2009)}

Voracek (2009) correlated annualized (2005-2007) total-population suicide rates for Italian regions with regional average scores from the 2006 PISA study (for general background of suicide 
prevalence in Italy, see Vichi, Masocco, Pompili, Lester, Tatarelli \& Vanacore, 2010). The latter variable, taken from Lynn (2010a), was available for 13 out of the 20 administrative regions of Italy. In the ecologic analysis, across the regions these two variables were correlated with Pearson $r=.57$ or Spearman $r_{\mathrm{s}}=.55$ (two-tailed $p$ values $=.04$ and .05 , respectively). Two new data sets, made accessible since then, now allow for independent replication attempts of this evidence.

First, Cornoldi, et al. (2010) published average scores for all 20 Italian regions from the very large and representative INVALSI (Istituto Nazionale per la Valutazione del Sistema Educativo di Istruzione e di Formazione) assessment. Utilizing the principle of reduction of measurement error sources through aggregation of parallel measurements, averaged scores across grades (2nd, 5th, and 8th) and domains (Italian and mathematics subtests) were used for the below analysis. The six available variables ( 3 grades x 2 domains) aggregated for analysis indeed showed high internal consistency (Cronbach $\alpha=$.88). Second, Lynn (2010b) reported mean regional results from a large, internet-based IQ assessment (www.sitozero.com). These data also appear in Templer (2012) and are available for 19 out of the 20 Italian regions (except for the Aosta Valley). Henceforth, for the sake of brevity, the above three data sources are referred to as PISA, INVALSI, and Internet IQ.

Calculated for the 13 regions with non-missing data in all three sources, internal consistency of the composite of PISA, INVALSI, and Internet IQ was high $(\alpha=.87)$. Accordingly, exploratory factor analysis (principal components method) extracted a single factor, with eigenvalue $\lambda=2.7$ and accounting for $90 \%$ of the variance in the data, thus suggesting factorial unidimensionality of the composite. Correlations between PISA and INVALSI, PISA and Internet IQ, and between INVALSI and Internet IQ were $.85, .87$, and .81 (all $p<.001)$. Coefficients of variation $(C V=S D / M)$ amounted to 0.054, 0.034, and 0.037 for PISA, INVALSI, and Internet IQ (based on 13, 20, and 19 regions, respectively), thus indicating broadly comparable distributional spread across the different data sources. 
As for the central analysis, INVALSI and Internet IQ were correlated with Spearman $r_{\mathrm{s}}=.56$ $(p=.01)$ and $.49(p=.03)$ with suicide rates across 19 out of 20 (INVALSI) or all 20 (Internet IQ) Italian regions. This means that the previous finding (Voracek, 2009), drawing on 13 regions with available data, were now replicated with two further, independent data sources, based on 19 or all 20 Italian regions. The above convergent and paralleling findings also speak to the representativeness and validity of the Internet IQ data source, which evidently was sufficient, as quite similar results were obtained vis-à-vis the very large and representative INVALSI assessment data.

Group-level effects from ecologic analyses, such as reported in Voracek (2009) and replicated here twice, may or may not generalize to effects on the individual level. The latter possibility is known as cross-level bias, i.e., drawing erroneous individual-level conclusions from group-level data. The only way to overcome this validity threat is to have individual-level data. In this context, it is interesting to note that the group-level finding of Voracek (2009) has meanwhile been directly confirmed by recent individual-level evidence. Pompili, Vichi, Qin, Innamorati, De Leo and Girardi (2013) reported a register-based study of all 2006-2008 deaths in Italy. Suicide victims had higher educational attainment than (same-sex and same age-bracket) deaths from all other causes. Subgroup analyses showed that this effect generalized to both sexes and all age bands (except for being attenuated and less obvious for old age) and was preserved with adjustments made for other variables, such as marital status and region of residence.

\section{CONCLUSION}

The novel, individual-level evidence of Pompili, et al. (2013) disconfirms all of Felice's (2010) concerns, alternative group-level analyses, and post-hoc conjectures vis-à-vis the original evidence of Voracek (2009). In Italy, higher educational attainment (a close proxy of psychometrically measured IQ; Deary, et al., 2007) corresponds to higher risk of suicide. This effect on the individual level is also reliably captured with aggregated data in ecologic (geographic) 
analyses. In conclusion, the novel lines of empirical evidence presented in this Responsum to Felice (2010) once again nicely demonstrate the truth and wisdom of the old dictum (ascribed to Goethe) that "data are the natural enemy of hypotheses" (Voracek \& Fisher, 2009). 


\section{REFERENCES}

BERALDO, S. (2010) Do differences in IQ predict Italian north-south differences in income? A methodological critique to Lynn. Intelligence, 38, 456-461.

Cornoldi, C., Belacchi, C., Giofrè, D., MARTini, A., \& Tressoldi, P. (2010) The mean southern Italian children IQ is not particularly low: a reply to R. Lynn (2010). Intelligence, 38, 462-470.

CORNOLDI, C., GIOFRÈ, D., \& MARTINI, A. (2013) Problems in deriving Italian regional differences in intelligence from 2009 PISA data. Intelligence, 41, 25-33.

DEARY, I. J. (2012) Intelligence. Annual Review of Psychology, 63, 453-482.

DeAry, I. J., Strand, S., SMith, P., \& FERnAndes, C. (2007) Intelligence and educational achievement. Intelligence, $35,13-21$.

Durkheim, É. (1897/1997) Suicide: a study in sociology. (J. A. Spaulding \& G. Simpson, Transl.) New York: Free Press.

ELLnER, M. (1977) Research of international suicide. International Journal of Social Psychiatry, 23, 187-194.

FELICE, E. (2010) Notes on the determinants of suicide rates in Italy's regions: a reply to Voracek (2009). Psychological Reports, 106, 731-736.

FELICE, E., \& GiUgliano, F. (2011) Myth and reality: a response to Lynn on the determinants of Italy's north-south imbalances. Intelligence, 39, 1-6.

HunT, E. B. (2011) Human intelligence. Cambridge: Cambridge Univer. Press.

KERKHOF, A., \& KUnST, A. (1994) A European perspective on suicidal behaviour. In R. Jenkins, S. Griffiths, I. Wylie, K. Hawton, G. Morgan, \& A. Tylee (Eds.), The prevention of suicide: a conference organised by the Department of Health, Faculty of Public Health Medicine, Royal College of General Practitioners and the Royal College of Psychiatrists. London: Her Majesty's Stationery (HMSO). Pp. 22-33. 
LYNN, R. (2010a) In Italy, north-south differences in IQ predict differences in income, education, infant mortality, stature, and literacy. Intelligence, 38, 93-100.

LYNN, R. (2010b) IQ differences between the north and south of Italy: a reply to Beraldo and Cornoldi, Belacchi, Giofre, Martini, and Tressoldi. Intelligence, 38, 451-455.

LYNN, R. (2012a) IQs in Italy are higher in the north: a reply to Felice and Guigliano. Intelligence, 40, 255-259.

LYNN, R. (2012b) North-south differences in Spain in IQ, educational attainment, per capita income, literacy, life expectancy and employment. Mankind Quarterly, 52, 265-291.

Morselli, H. (1881) Suicide: an essay on comparative moral statistics. London: C. Kegal Paul.

Plomin, R., DeFries, J. C., McClearn, G. E., \& McGuffin, P. (2008) Behavioral genetics (5th ed.). New York: Worth.

Pompili, M., Vichi, M., Qin, P., InNAmorati, M., DE LeO, D., \& Girardi, P. (2013) Does the level of education influence completed suicide? A nationwide register study. Journal of Affective Disorders, 147, 437-440.

RindermanN, H., WoOdLEY, M. A., \& STRATFORD, J. (2012) Haplogroups as evolutionary markers of cognitive ability. Intelligence, 40, 362-375.

SNYDERMAN, M., \& RothMAN, S. (1987) Survey of expert opinion on intelligence and aptitude testing. American Psychologist, 42, 137-144 .

SNYDERMAN, M., \& Rothman, S. (1990) The IQ controversy, the media, and public policy. New Brunswick, NJ: Transaction Publ.

TEMPLER, D. I. (2012) Biological correlates of northern-southern Italy differences in IQ. Intelligence, 40, 511-517.

Vichi, M., MAsocco, M., POMPILI, M., Lester, D., TATARELli, R., \& VANACORE, N. (2010) Suicide mortality in Italy from 1980 to 2002. Psychiatry Research, 175, 89-97. 
VORACEK, M. (2005) The social ecology of intelligence and suicide in Belarus. Journal of Social Psychology, 145, 613-617.

VorACEK, M. (2006a) Regional intelligence and suicide rate in Denmark. Psychological Reports, 98, 671-674.

VoraceK, M. (2006b) Smart and suicidal? The social ecology of intelligence and suicide in Austria. Death Studies, 30, 471-485.

VoraceK, M. (2009) Regional intelligence and suicide rate in Italy. Psychological Reports, 105, 10231026.

VORACEK, M., \& FISHER, M. L. (2009) Data are the natural enemy of hypotheses: reply to Holland (2009). Archives of Sexual Behavior, 38, 460-462.

WAINER, H. (1989) Responsum. Journal of Educational Statistics, 14, 187-199. 